\title{
Metal spherical nanostructures and dielectric response quantum-size effects in silver nanoclusters
}

\author{
Koray KÖKSAL a, $\nabla$, Yaroslav PAVLYUKH ${ }^{\text {b }}$, Jamal BERAKDAR ${ }^{\text {b }}$ \\ a Bitlis Eren University, Faculty of Art and Science, Department of Physics, TR 13000, Bitlis-Turkey \\ ${ }^{b}$ Martin Luther University, Department of Physics, D 06120, Halle-Germany \\ $\checkmark$ Corresponding author: Tel; +49 34555285 25, fax; +49345 55273 93, e-mail: kkoksal@beu.edu.tr
}

\begin{abstract}
The change of the quantum dielectric response of metal clusters has been investigated by tuning their size and topology. In order to calculate the change, analytical and numerical methods have been used. Results are presented for silver metal spheres. We have discussed possible ways for the experimental verification on the basis of scanning tunneling spectroscopy (STM) (Limot et al. 2005) or electron energy loss spectroscopy (EELS) (Eccles et al. 2010).
\end{abstract}

Keywords: Dielectric function, electronic structure, nanoclustes, surface states

\section{Introduction}

Recently, metallic nanoclusters have received great interest due to their electronic and optical properties which are strongly dependent on the cluster size (Wei et al. 2011). They are good candidates for biological (Shanga et al. 2011), biomedical (Maity et al. 2011) devices, optics, optoelectronics and plasmonics (Wei 2011). Metal nanoclusters show quantum-size effects if they are sufficiently small. Nowadays, these effects and related properties can be observed by using state-of-art experimental devices like STM and EELS.

In order to reveal the electronic properties of the metal nanostructures, some theoretical techniques have been developed for different metal nanostructures (Piskunov et al. 2011; Zhang et al. 2011). As a difference from bulk materials, the nanoclusters have some additional states like surface and image states which are currently investigated by high technological experimental devices (Tusche et al. 2011; Ünal et al. 2011). The optical properties of a metal cluster are strongly dependent on its electronic properties. Although these properties have been investigated theoretically in literature, classical approximation rather than quantum mechanics have been used to obtain electronic and optical characteristics of the nanostructures.

In this study, the first aim is to introduce a quantum mechanical description of a small quantum system which is defined by a spherical symmetric metal sphere. The second aim is to calculate the microscopic dielectric response of this system to an external applied field as a function of the radial position of the sphere. As a third aim, we try to explain the relationship between our results and recent experiments. The rest of the paper is organized as follows: In the next section, the theoretical models are presented. After that, the results are showed and detailed discussions are given. In the last section, a brief conclusion is presented.

\section{Theoretical Models}

\subsection{Semi-empirical potential for definition of surface structure}

Because we define the system as a spherical symmetric cluster, it can be said that the electrons make an angular motion freely along the surface and parallel layers. This angular motion is described by Spherical Harmonics. The only electronic potential which the electrons feel is the radial semi-empirical potential which is obtained by using empirical techniques and ab-initio methods. One can see the shape of this radial potential and representative shape of the metal cluster in Figure 1.

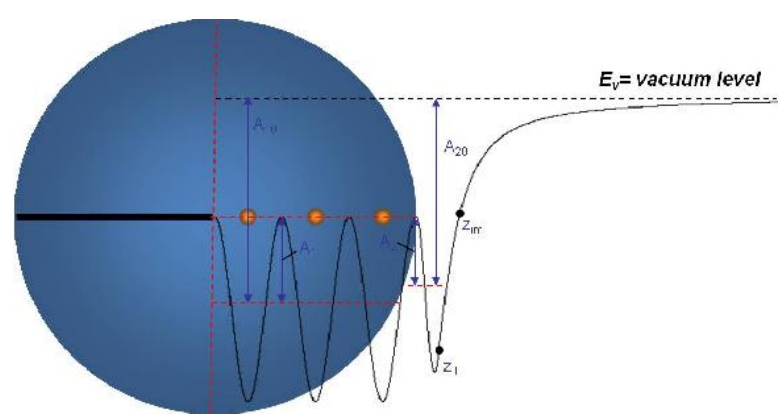

Figure 1. Shape of the structure and radial potential which is used in the calculations of the electronic structure and optical properties. Orange dots indicate one atom of the layer. The parameters are taken from the Chulkov's study (Chulkov et al. 1999). 
As can be seen in Figure 1, the source of the potential oscillation is the presence of the atomic layers. Every orange dot indicates an atomic layer. In the center of the sphere, the potential is zero because there is no atom. When approaching the position of the atom, the potential is decreasing and it has a minimum value at the place of the atom. The potential oscillation is terminated on the surface. The potential curve in the vacuum region is modelled to produce surface and image electron states.

\subsection{Electronic structure of spherical cluster}

Because of the spherical symmetry, the wavefunction of a single electron can be written as

$$
\psi_{n \ell m}(r, \theta, \varphi)=R_{n \ell}(r) \mathrm{Y}_{\ell m}(\theta, \varphi)
$$

where $Y_{\ell m}$ is the spherical harmonics; $n, \ell, m$ are the discrete quantum numbers. In order to obtain the radial wavefunctionof a single electron, it is enough to solve 1D Schrodinger's Equation as

$$
H \psi_{n \ell m}(r, \theta, \varphi)=E \psi_{n \ell m}(r, \theta, \varphi)
$$

which has a more explicit form

$$
-\frac{\nabla^{2} \psi_{n \ell m}}{2}+V(r) \psi_{n \ell m}=E \psi_{n \ell m}
$$

where it is assumed that $\hbar=\mathrm{m}=\mathrm{e}=1$. In order to calculate the energy eigenvalues and eigenfunctions, numerical shooting method and four-step Runga Kutta techniques are used.

\subsection{Formalism of dielectric response function}

The dielectric response is a very important quantuity because it provides valuable information about the main optical properties of the structure. The dielectric response is dependent on the electronic structure of the system as well as the interaction between the electrons. The frequency of the external applied field is the relevant parameter.

The explicit form of the dielectric response function is

$\varepsilon\left(r, r^{\prime \prime}, \omega\right)=\delta\left(r, r^{\prime \prime}\right)-$

$\sum_{n, \ell, n^{\prime}, \ell^{\prime}} \frac{f_{n, \ell^{-}-f_{n^{\prime}, \ell^{\prime}}}}{E_{n, l}-E_{n^{\prime}, l^{\prime}}-\omega-i \eta} \psi(r) \psi(r)^{*} \int \frac{\psi\left(r^{\prime}\right) \psi\left(\mathbf{r}^{\prime}\right)^{*}}{\left|r-r^{\prime}\right|} d r^{\iota}$

where $\eta$ is infinitesimal number to avoid the singularity in the eqaution. $E_{n, \ell}$ and $E_{n^{\prime}, \ell^{\prime}}$ are discrete energy states because of the radial confinement. $f_{n, \ell}$ and $f_{n^{\prime}, \ell^{\prime}}$ are fermi distribution function. This form is obtained by the random phase approximation. The term $1 /\left|r-r^{l}\right|$ is the coulomb interaction beween the electrons.

\section{Results and Discussion}

The electronic structure of a silver cluster can be seen in Figure 2 in detail. The radial potential and corresponding energy levels have been calculated by using the techniques which are mentioned in previous section. The radius of the silver cluster is 4.43 a.u. As can be seen in the Figure 2, the potential is dependent on the radial position as well as the orbital quantum number, $\ell$. So far, the radial potential is consturcuted as to deliver the conventional surface structure in the planar case (Winkelmann et al. 2007). For $\ell=0$, there are two electron levels below fermi energy, for $\ell>0$ there is one level, for $\ell=4$ there is no energy level. In Figure 2, the energy states which are above fermi energy and below vacuum level are named as surface states. These states are localized on the surface or near the surface.

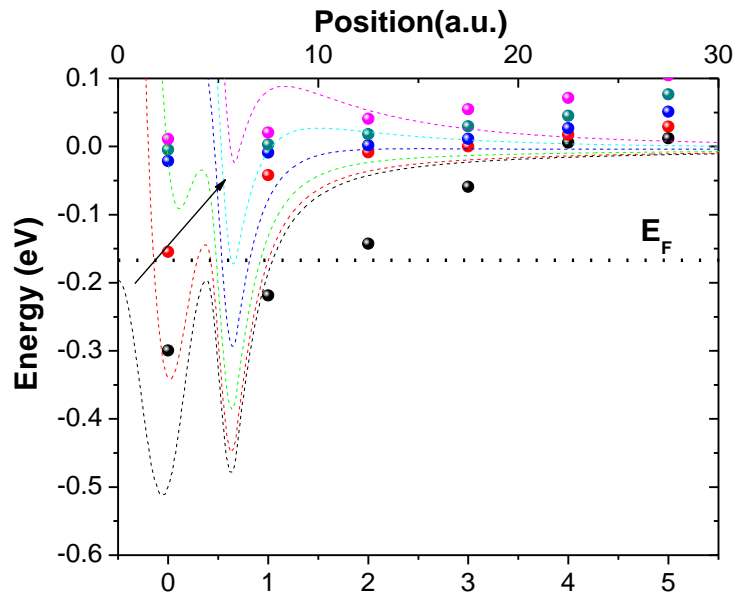

Figure 2. The dots show the discrete energy levels versus orbital quantum numbers $\ell$ for a nanocluster which has a 4.43 a.u. radius. Dotted lines indicate the variation of the potential along radius for different $\ell$ values. Black line is for $\ell=0$, purple line is for $\ell=4$

For this structure, the probability distribution of the bulk, surface and image state electrons can be seen in Figure 3. As seen in the Figure 3, while bulk electrons are localized inside the sphere, surface and image states mostly localized on and out of the surface. One should notice that the surface and the image states appear due to the termination of the crystal structure. Surface properties and the dynamics are determined by these electronic states. When the size of the system is decreased to the nano scale, the confinement effects begin to emerge. The continuous energy distribution is converted to discrete energy levels. The possible optical transtions between the states are limited.

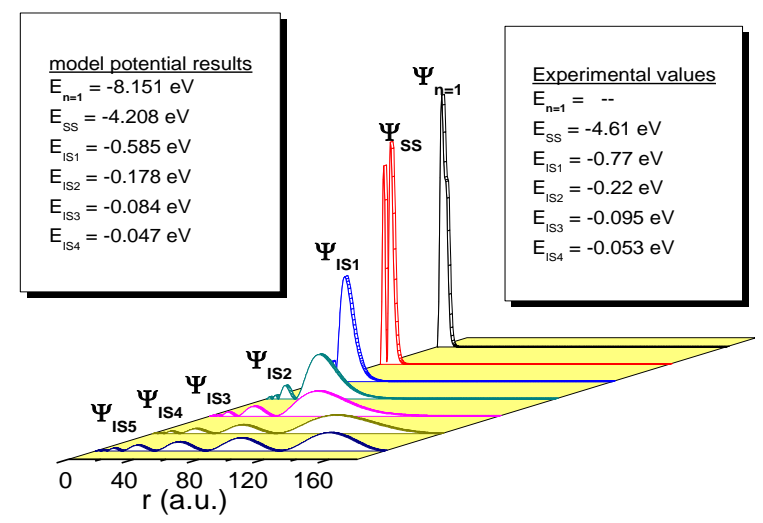

Figure 3. Bulk, surface and image states of the silver cluster which has a 4.43 a.u. radius. On the inset boxes, there is a comparison of the experimental and our results. 
In the frame of the discussion about surface states and confinement effect, we can explain the result for position dependent dielectric response function which is shown in the Figure 4. For $z=\theta=0$, the real part dielectric function changes with the variation of $z^{\prime \prime}$ and $\omega$. The primary effect of the increase in $z^{\prime \prime}$ is the decrease in amplitude of the dielectric function. The function have the peaks for different $\omega$ values which show the transitions between electron states. Some of these transitions occur between bulk and surface states. The dependency of the dielectric response on the position means that the dielectric response gives different values everywhere on the structure. This is the result of the change in the electron density along the radial position.

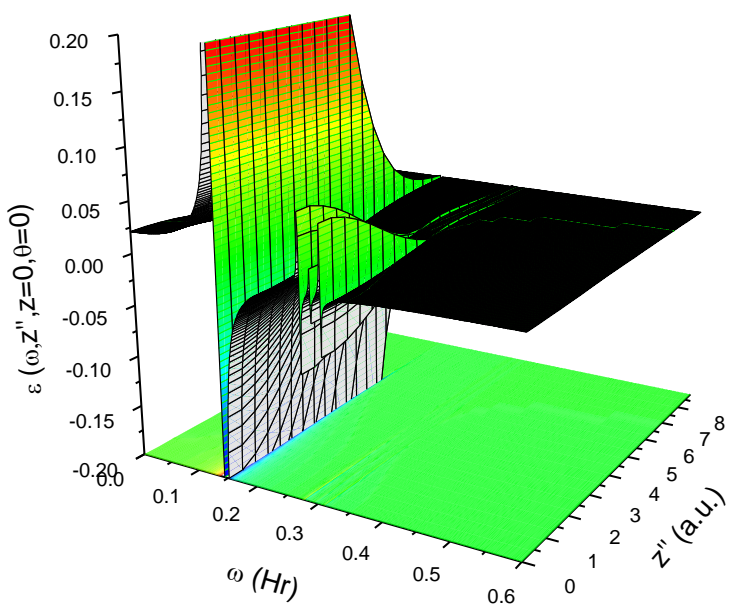

Figure 2. The change of real part of the dielectric response function with respect to radial position and frequency. In the center of the sphere, while the amplitude of the response has maximum value, this value is decreasing with approaching to the surface. The sharp peaks along the frequency axis show the optical transitions between the electronic states.

\section{Conclusion}

The investigation of the dielectric response function in small metal clusters is very important with respect to technological applications. The relationship between bulk, surface and confinement states can provide valuable information about the more complicated observed phenomena. In our study, calculating the electronic structure and the position dependent dielectric function, we aimed to expose the fundamental reasons of the more complicated results which are obtained for small metal clusters. Another important result is the dependency of the dielectric response on the position.

\section{References}

Limot L, Pehlke E, Kröger J, Berndt R (2005). Surfacestate localization at adatoms. Phys Rev Lett 94, 036805.

Eccles JWL, Bangert U, Bromfield M, Christian P, Harvey AJ, Thomas P (2010). UV-Vis plasmon studies of metal nanoparticles. J Phys Conf Ser 241, 012090.

Chulkov EV, Silkin VM, Echenique PM (1999). Image potential states on metal surfaces: binding energies and wave functions. Surf Sci 437, 330-352.

Maity D, Chandrasekharan P, Pradhan P, Chuang KH, Xue JM, Feng SS, Ding J (2011). Novel synthesis of superparamagnetic magnetite nanoclusters for biomedical applications. J Mater Chem 21, 1471714724.

Piskunov S, Zvejnieks G, Zhukovskii YF, Bellucci S (2011). Atomic and electronic structure of both perfect and nanostructured Ni (111) surfaces: first-principles calculations. Thin Solid Films 519, 3745-3751.

Shanga L, Dong S, Nienhaus UG (2011). Ultra-small fluorescent metal nanoclusters: synthesis and biological applications. Nano Today 6, 401-418.

Tusche C, Ellguth M, Ünal AA, Chiang CT, Winkelmann A, Krasyuk A, Hahn M, Schönhense G, Kirschner J (2011). Spin resolved photoelectron microscopy using a twodimensional spin-polarizing electron mirror. Appl Phys Lett 99, 032505-032507.

Ünal AA, Tusche C, Ouazi S, Wedekind S, Chiang CT, Winkelmann A, Sander D, Henk J, Kirschner J (2011). Hybridization between the unoccupied shockley surface state and bulk electronic states on $\mathrm{Cu}$ (111). Phys Rev B 84, 073107/1-4.

Wei H (2011). Plasmonic silver nanoparticles for energy and optoelectronic applications, Wiley.

Wei W, Lu Y, Chen W, Chen S (2011). One-pot synthesis, photoluminescence, and electrocatalytic properties of subnanometer-sized copper clusters. J Am Chem Soc 133, 2060-2063.

Winkelmann A, Bisio F, Ocaña R, Lin W C, Nývlt M, Petek H, Kirschner J (2007). Ultrafast optical spin injection into image-potential states of $\mathrm{Cu}$ (001). Phys Rev Lett 98, 226-601.

Zhang XD, Guo ML, Wu D, Liu PX, Sun YM, Zhang LA, She Y, Liu QF, Fan FY (2011). First-principles investigation of ag-doped gold nanoclusters. Int J Mol Sci 12, 29722981. 